\title{
Effects of corrective exercises on selective functional movement assessment and health risk appraisal in middle-aged women
}

\author{
Jae Eun Kim ${ }^{\mathrm{a}}$, Cheong Kim ${ }^{\mathrm{b}}$, Sinseop Kim ${ }^{\mathrm{c}}$ \\ ${ }^{a}$ Specialized Physical Therapy Team, Barosun Hospital, Seoul, Republic of Korea \\ ${ }^{b}$ Department of Health Management, College of Health and Welfare, Sahmyook University, Seoul, Republic of Korea \\ ${ }^{c}$ Department of Counseling Psychology, College of Health Science and Social Welfare, Sahmyook University, Seoul, Republic of Korea
}

Objective: The purpose of this study was to find the limited patterns of middle-aged women in selective functional movement evaluation and analyze the effects of pattern improvement exercises and general control groups on the Health Risk Appraisal (HRA).

Design: Randomized controlled trial.

Methods: The 31 subjects were physically healthy middle-aged women aged 40-59 living in Seoul, The subjects were randomly divided into an experimental group and a control group. Forty-three physically healthy women were originally recruited and randomly assigned to either the experimental group $(n=22)$ or the control group $(n=21)$. However, due to lack of participation, a total of seventeen subjects in the exercise group and fourteen subjects in the control group participated in the study. All subjects were tested using Selective Functional Movement Assessment (SFMA) and HRA for the baseline measurement and joined an exercise program of their group for one hour per session, twice a week for four weeks. The experimental group was provided with the corrective exercises and the control group was given the general fitness program. A follow-up test was conducted after eight weeks from the baseline measurement.

Results: Both experimental and control group showed significant changes in SFMA and HRA scores $(p<0.05)$. In the experimental group and control groups, the SFMA and HRA showed significant improvement from baseline to 4 weeks $(p<0.05)$. Also, in the experimental group, the SFMA was significantly improved from baseline to 8 weeks $(p<0.05)$. For the experimental group, there was a significant improvement in SFMA after 4 weeks compared to the control group $(p<0.05)$.

Conclusions: The corrective exercise in the experimental group had a positive effect on the SFMA score as well as the general squat exercise in the control group. The corrective exercise and general control group had the same positive effect on the HRA score.

Key Words: Corrective exercise, Health Risk Appraisal, Middle-aged women, Selective Functional Movement Assessment

\section{Introduction}

Performing regular exercises is essential for middle-aged women to improve their health. Middle-aged women experience hormone change physiologically [1] and consequently have various symptoms such as facial blushing, headache, urinary frequency, urogenital organ disorder, musculoskeletal pain, depression, and forgetfulness [2]. There have been many studies on exercise to improve and keep middle-aged women's health. With regard to domestic studies on exercise intervention, there are studies on aerobic exercise and complex exercise [3], complex exercise [4], re-

Received: 7 November, 2016 Revised: 12 December, 2016 Accepted: 13 December, 2016

Corresponding author: Sinseop Kim

Department of Counseling Psychology, College of Health Science and Social Welfare, Sahmyook University, 815 Hwarang-ro, Nowon-gu, Seoul 01795, Republic of Korea

Tel: 82-2-3399-1637 Fax: 82-2-3399-1680 E-mail: kimss36@syu.ac kr

(c) This is an Open-Access article distributed under the terms of the Creative Commons Attribution Non-Commercial License (http://creativecommons.org/licens es/by-nc/4.0) which permits unrestricted non-commercial use, distribution, and reproduction in any medium, provided the original work is properly cited.

Copyright $\odot 2016$ Korean Academy of Physical Therapy Rehabilitation Science 
sistance exercise [5] and circulation exercise [6]. Therefore, it has been found that doing exercises is required for middle-aged women's health.

However, all exercises do not bring about the same effects, as shown in group exercise [7], resistance and aerobic exercises [8], and exercises focusing on particular diseases [9]. No matter how good the exercises are, doing exercises without any consideration of individuals' body conditions excessively increases physical activity load and may increases the risk of damage [10].

Recently, there have been active research in which customized exercises are effective [11]. The purpose of customized exercises is to provide proper exercises in consideration of individuals' health information, diseases, and physical strength [12], to change personal behavior and recover physical ability, physical strength, and health [13].

An assessment tool called Selective Functional Movement Assessment (SFMA) has been developed with the concept that there is functional connection with stability and mobility of the body segment in which an exercise program can be customized for the individual. With this tool, individuals are able to find their physically limited patterns. Functional corrective exercises are referred to as exercise to correct the repeated physical patterns in daily living [14].

We used the Health Risk Appraisal (HRA) questionnaire which is Life Transition Period Health Examination developed by Korea Association of Health Promotion to evaluate Korean women in their 40s. In the HRA questionnaire, smoking, drinking, exercise, nutrition, and obesity were asked to assess health status [15]. Researches were conducted on the effects of various exercises on HRA, and on the correlation between exercise amount and HRA. There are studies on grasping power [16], on walking speed [17], on muscle force [18], and on balance ability [19]. As such, many studies focused on HRA through physical ability and revealed the effects of physical ability and exercises on health.

We used the HRA questionnaire which is Life Transition Period Health Examination developed by Korea Association of Health Promotion to evaluate Korean women in their 40s. In the HRA questionnaire, smoking, drinking, exercise, nutrition, and obesity were asked to assess health status [15].

For smoking, Fagerstrom Test for Nicotine Dependence Korea version (FTND-K) whose reliability and validity were already proved was applied. In each item, participants were allowed to select proper items of smoking habits. The inter-rater reliability was 0.69 , and the test-retest reliability was 0.88 [20]. For drinking, Alcohol Use Disorders Identification Test (AUDIT-K) whose reliability and validity were already proved was applied for participants to make self-diagnosis of their drinking habits and dependence. The inter-rater reliability was 0.92 , and the test-retest reliability was 0.86 [21]. For exercise, the test tool is comprised of three questions from the Physical Activity Assessment Questionnaire of Lifescripts Guide [22] and 7 questions from the Physical Activity Readiness Questionnaire (PAR-Q). The inter-rater reliability was 0.92 [23]. For nutrition, mini dietary assessment index (MDA) made up of 10 questions was used (5 Point in each question for a total score of 50 scores). In each question, participants are able to select 1, 3, or 5 scores depending on their dietary habits [24]. This study used 11 questions of the new version of the secondary Life-Transition-Period Health Diagnosis. For obesity, the tool has three translated questions of Lifescripts Guide and the question about waist size and body mass index (BMI) [22].

Group exercises face limits to customized exercises. A group exercise has been generalized, and has become the first exercise that many people experience. There is a lack of research on the exercises that cause no side effects on middle-aged women that are helpful for HRA. If as many patterns movement activities are applied to a group exercise, it will be more effective than a simple-pattern exercise. This study tries to apply a simple pattern exercise and multiple-pattern exercise to middle-aged women to investigate whether SFMA score is improved and how the exercises influence HRA.

\section{Methods}

\section{Subjects}

Forty three middle-aged women subjects (Figure 1) were recruited by poster announcement and word of mouth. The study subjects were physically healthy middle-aged women aged 40-59 living in Seoul, who understood the purpose of this study and agree to participate in this experimental research in writing. Prior to participation, subject's signed an informed consent form approved by the Institutional Review Board (SYUIRB2015-105). The subjects were randomly divided into an experimental group and a control group. The subjects in both groups were tested using SFMA and HRA questionnaire. All subjects joined an exercise program of their group one hour per session, twice a week for four weeks. Of the 43 study subjects, the experimental group had 

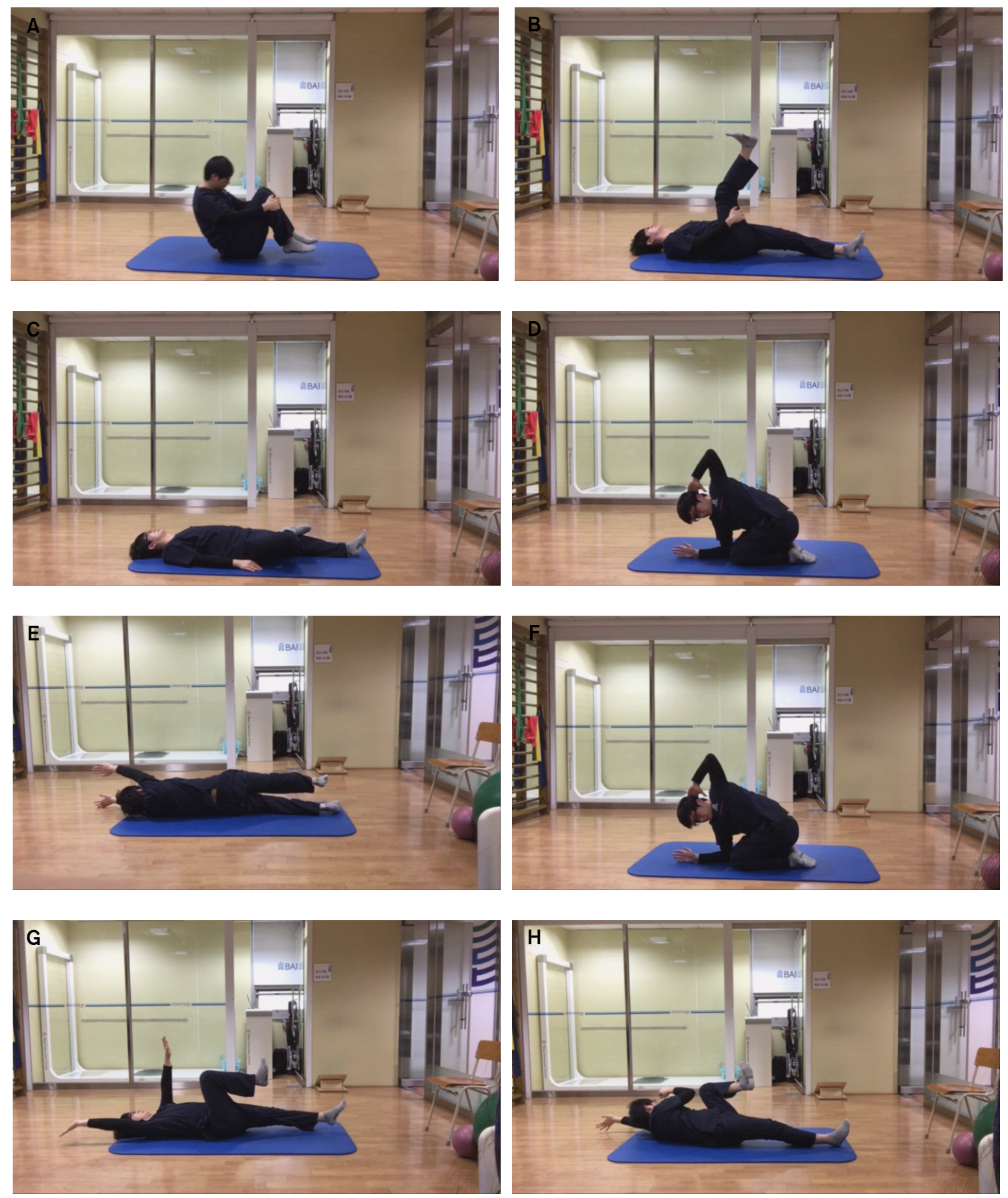

Figure 1. Corrective exercise. (A) Flexion pattern 1: Roll the knees to the chest tightly and roll back and forth like a ball. (B) Flexion pattern 2: In the supine position, lift one leg and put the hands on back of the knee for the stretch. (C) Extension pattern 1: In the supine position, lift your one leg and put it on the knee of the other leg, and stretch it out. (D) Extension pattern 2: In kneeling position, place both elbows in front of the knees. Put one arm on the behind your head. Turn your trunk and look up with the arm pointing to the ceiling. (E) Rotation pattern 1: In the supine position, reach out both arms. Stretching with one leg, roll into prone position. And using same leg back and return to the supine position. (F) Rotation pattern 2: In kneeling position, place both elbows in front of the knees. Put one arm on the behind your head. Turn your trunk and look up with the arm pointing to the ceiling. (G) Single leg stance pattern 1: In the supine position, put one arm and one leg on the other side on the floor. $(\mathrm{H})$ Single leg stance pattern 2: Make the other arm and leg contact and then roll left and right slowly. (I) Overhead deep squat pattern 1: In the crawling posture on all fours, tighten your stomach and keep pushing the floor with the legs. Go forward in the position. (J) Overhead deep squat pattern 2: In kneeling position, place both elbows in front of the knees. Put one arm on the behind your head. Turn your trunk and look up with the arm pointing to the ceiling. 

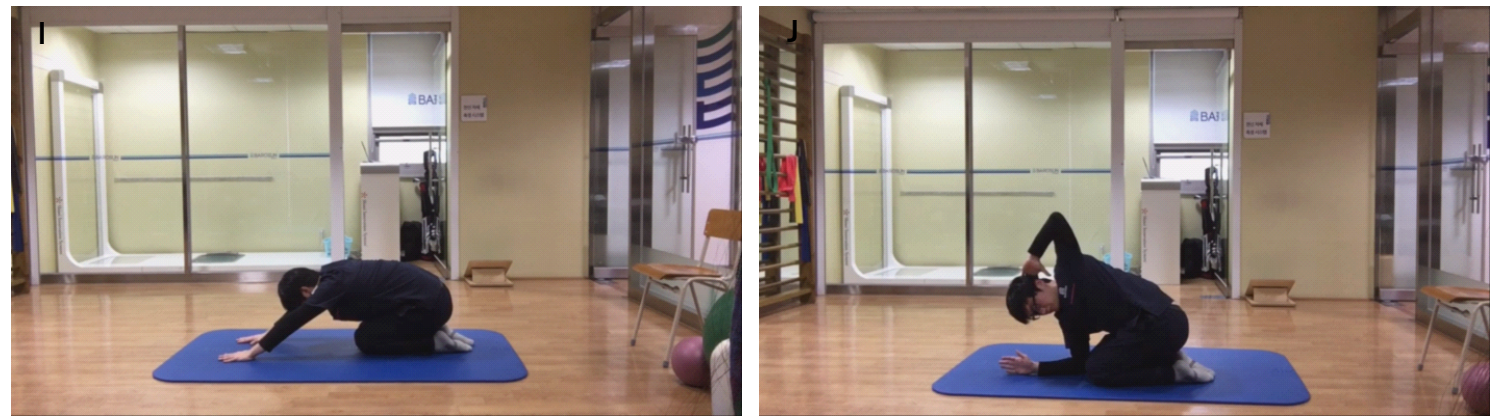

Figure 1. Continued.

22 women and the control group had 21 . Because 12 subjects were absent for more than three sessions during the exercise program, they were excluded from the study. As a result, the experimental group of 16 subjects and the control group of 14 subjects participated in the post-test. One month later, a follow-up test was conducted to measure of their test scores have been maintained.

\section{Intervention}

In both experimental and control groups, a main exercise program was provided. The main exercise programs were different between the two groups. The exercise program was designed with a warm-up and cool-down session before and after the main exercises. The amount of time for warm-up and cool-down required about 10 minutes to complete and the same protocols were used for both groups. The protocol for warm-up and cool-down was designed by the American Heart Association.

The experimental group was provided with the corrective exercises with the movement pattern that was most dysfunctional to each subject. The control group was given the general fitness program mostly used in the average gym.

For the corrective exercises group, two main different types of exercise were used $4 \times 4$ Matrix Exercise and individualized exercise. During the first half of the corrective exercises, 4×4 Matrix Exercise (http://www.mytpi.com/exercises \#4x4_matrix) was performed. Each of the 12 movements in the $4 \times 4$ Matrix was repeated four times.

During the second half of the corrective exercises, two functional exercises for the first two most dysfunctional movement patterns were given to each subject. Most dysfunctional movement pattern was selected based on the result of the SFMA test. For each movement pattern two corrective exercises were applied (Figure 1). Each subject was instructed to perform two corrective exercises for 4 weeks.
On the first week, subjects were told to perform 6 repetitions per exercise. Each week, 2 repetitions were added for both exercises they were performing. On the last week, subjects performed 12 repetitions per exercise.

For the control group, the trainers instructed a squat exercise to the subjects. Squat, dead lift and bench press are three main motions used in the general fitness program in the average gym. Squat is a very common movement to improve essential muscles such as quadriceps femoris muscle, hamstring, gluteus maximus, and erector spinae muscle, and is very effective in correcting a posture through precise movement. In consideration of the study subjects' age and exercise level, this study performed a step-by-step squat and added different exercise strength depending on individuals' level.

\section{Measurement}

\section{SFMA}

To assess a person's functional movement level, each participant was tested with SFMA, which consisted of seven movement patterns: 1) cervical movement pattern, 2) upper extremity pattern, 3) multi-segmental flexion, 4) multi-segmental extension, 5) multi-segmental rotation, 6) single leg balance, and 7) overhead deep squat. All movement patterns were performed bilaterally when applicable. Applying the series of tests in seven movement patterns to a patient provides information to determine if a movements pattern is functional or dysfunctional [25]. Recent studies have shown that good diagnostic reliability can be achieved when applied SFMA to patients without musculoskeletal disease. Regarding the internal reliability of SFMA, Kappa Coefficient of three evaluators who had almost perfect consistent points of $0.72-0.83$, and a consistency rate of $83 \%-88 \%$ [26]. The total score of SFMA top tier test (SFMA score) is used to determine the difference of functional level before and af- 
ter the intervention. The results each of the five movement patterns in the SFMA test were used to determine the most dysfunctional movement pattern. The first two most dysfunctional patterns were determined and two corrective exercises for each pattern were used for the main exercise. The cervical movement pattern and upper extremity pattern was excluded from the exercise consideration because it does not include spinal or trunk movement.

\section{HRA}

HRA is Life Transition Period Health Examination developed by Korea Association of Health Promotion to evaluate Koreans in their 40s. Smoking, drinking, exercise, nutrition, and obesity were asked to assess health status. In the HRA questionnaire each assessment tool can determine dependency or health status based on the scores. All scores were combined and analyzed for each subjects.

\section{Statistics}

For statistical analysis, this study used IBM SPSS Statistics ver. 19.0 (IBM Co., Armonk, NY, USA). After normality test, the result met the assumption of normal distribution, and thereby homogeneity was secured through t-test prior to intervention. To draw a comparison on the effects of interventions between the experimental group and the control group, repeated measured ANOVA and t-test were conducted. To compare the effects of intervention time

Table 1. General characteristics

$(\mathrm{N}=31)$

\begin{tabular}{lll}
\hline \multicolumn{1}{c}{ Characteristic } & $\begin{array}{l}\text { Experimental } \\
\text { group }(\mathrm{n}=17)\end{array}$ & $\begin{array}{c}\text { Control group } \\
(\mathrm{n}=14)\end{array}$ \\
\hline Height $(\mathrm{cm})$ & $159.5(0.044)$ & $160.7(0.057)$ \\
Weight $(\mathrm{kg})$ & $58.76(5.89)$ & $57.04(9.67)$ \\
Body mass index $\left(\mathrm{kg} / \mathrm{m}^{2}\right)$ & $23.04(1.87)$ & $21.96(2.17)$ \\
\hline
\end{tabular}

Values are presented as mean (SD). difference between the experimental group and the control group, Bonferroni post analysis was conducted. For the analysis on statistical significance, a significance level was set to $p<0.05$.

\section{Results}

\section{General characteristics}

Regard to the general characteristics, the experimental group and the control group were homogenous. The average height for the experimental group was measured at $159.5 \mathrm{~cm}$ and $160.7 \mathrm{~cm}$ for the control group. The average weight for the experimental group was measured $58.76 \mathrm{~kg}$, and the control group $57.04 \mathrm{~kg}$. The average BMI measured to be 23.04 $\mathrm{kg} / \mathrm{m}^{2}$ for the experimental group and $21.96 \mathrm{~kg} / \mathrm{m}^{2}$ for the control group (Table 1).

\section{The changes in SFMA and HRA scores}

Both experimental and control group showed significant changes in SFMA and HRA scores. There was a significant change in the SFMA score for the corrective exercise baseline to 4 weeks and baseline to 8 weeks. One-way repeated ANOVA showed the experimental group had $\mathrm{F}=29.393$ and $p<0.001$ (Table 2). There was significant change in HRA score for corrective exercise group from 4 weeks to 8 weeks after baseline assessment. One-way repeated ANOVA showed the experimental group had $\mathrm{F}=5.733$ and $p=0.007$ (Table 2).

Individuals who participated in the general control group also increased their scores on the SFMA from baseline to 4 weeks and 8 weeks baseline to repeated measures ANOVA for the control group was $\mathrm{F}=5.617$ and $p<0.001$ (Table 2). This same pattern of findings was found on the HRA for the control group of $\mathrm{F}=28.966$ and $p<0.001$ (Table 2).

To find the difference in the time-based effects between the experimental group and the control group on the basis of

Table 2. SFMA and HRA scores

$(\mathrm{N}=31)$

\begin{tabular}{|c|c|c|c|c|c|c|c|c|}
\hline \multirow{2}{*}{ Outcomes } & \multicolumn{4}{|c|}{ Experimental group $(n=17)$} & \multicolumn{4}{|c|}{ Control group $(n=14)$} \\
\hline & Baseline & $4 \mathrm{wk}$ & $8 \mathrm{wk}$ & $\mathrm{F}$ & Baseline & $4 \mathrm{wk}$ & $8 \mathrm{wk}$ & $\mathrm{F}$ \\
\hline SFMA (score) & $27.65(5.862)$ & $36.00(5.489)^{\mathrm{a}}$ & $36.53(6.866)^{\mathrm{a}}$ & $29.393^{*}$ & $30.71(5.525)$ & $35.50(5.317)^{\mathrm{a}}$ & $36.92(5.024)$ & $5.617^{*}$ \\
\hline HRA (score) & $20.35(5.049)$ & $23.18(5.446)^{\mathrm{a}}$ & $21.00(3.708)$ & $5.733^{*}$ & $17.43(5.667)$ & $22.54(4.789)^{\mathrm{a}}$ & $19.23(6.274)$ & $28.966^{*}$ \\
\hline
\end{tabular}

Values are presented as mean (SD).

SFMA: Selective Functional Movement Assessment, HRA: Health Risk Appraisal.

${ }^{a}$ Significant difference compared with baseline. $p<0.05$. 
Table 3. Comparison of SFMA and HRA between the two groups $(\mathrm{N}=31)$

\begin{tabular}{lcccccccc}
\hline \multirow{2}{*}{ Outcomes } & \multicolumn{3}{c}{ Baseline-4 wk } & & \multicolumn{3}{c}{ Baseline-8 wk } \\
\cline { 2 - 3 } & $\begin{array}{c}\text { Experimental group } \\
(\mathrm{n}=17)\end{array}$ & $\begin{array}{c}\text { Control group } \\
(\mathrm{n}=14)\end{array}$ & $\mathrm{t}$ & & $\begin{array}{c}\text { Experimental group } \\
(\mathrm{n}=17)\end{array}$ & $\begin{array}{c}\text { Control group } \\
(\mathrm{n}=14)\end{array}$ & $\mathrm{t}$ \\
\hline SFMA (score) & $8.35(4.372)$ & $4.79(3.786)$ & $2.399^{*}$ & & $8.88(5.776)$ & $3.57(9.874)$ & 1.867 \\
HRA (score) & $2.82(3.238)$ & $5.21(4.117)$ & -1.800 & & $0.65(3.534)$ & $0.43(9.517)$ & 0.88 \\
\hline
\end{tabular}

Values are presented as mean (SD).

SFMA: Selective Functional Movement Assessment, HRA: Health Risk Appraisal.

${ }^{*}$ Change in SFMA score from baseline to 4 weeks between the experimental group and control group $(p<0.05)$.

SFMA scores, Post-hoc was used. There were significant changes in SFMA scores from baseline to 4 weeks $(p<0.05)$ and baseline to 8 weeks $(p<0.05)$. There were no significant changes in HRA score for this experimental group.

Individuals who participated in the control group increased their scores on the SFMA from baseline to 4 weeks $(p<0.05)$, but there were no significant changes from baseline to 8 weeks. There was significant change in the HRA control group scores was from baseline to 4 weeks $(p<0.05)$. But no significant changes were found in the HRA control group score from baseline to 8 weeks.

\section{Comparison of SFMA and HRA scores between the two groups}

To find the difference in SFMA and HRA scores between the experimental group and the control group an independent t-test was conducted. The difference in SFMA scores from baseline to 4 weeks between the two groups were statistically and significantly different $(p=0.023, \mathrm{t}=$ 2.399; Table 3). However, the differences in SFMA scores from baseline to 8 weeks between the two groups were not significant.

The difference in HRA score between the two groups from baseline to 4 weeks did not show a significant difference. Also, the HRA scores between the two groups and from baseline to 8 weeks were not significant.

\section{Discussion}

This experiment was conducted to investigate how the corrective exercise and general control groups influence SFMA and HRA score. The SFMA score represents overall functional movement status and HRA represents current lifestyle of an individual.

Both experimental and control group showed significant changes in SFMA scores. The corrective exercise in the experimental group has positive effect on the SFMA score as well as general squat exercise in the control group. The warm-up and cool-down portion of both exercise programs were the same. The squat movement pattern was a major pattern used in the control group. But this pattern is one of the five movement pattern which was used to determine the type of exercise if the squat pattern was most dysfunctional. There are many similarities in both exercise programs.

The corrective exercise and general control group has same positive effects on the HRA score. In the HRA questionnaire, smoking, drinking, exercise, nutrition, and obesity were asked to assess health status. We can speculate that the exercise variable included in the questionnaire may have somewhat been affected by the exercise program.

The HRA includes many lifestyle behaviors. Previous studies revealed that the correction of habituated behavior directly influenced the function of the brain. It was reported that the 14-day short-term intervention including diet, relaxation exercise, cardiovascular conditioning, and mental exercise positively influenced brain metabolism and cognition function [27]. In 2011, the American Psychological Association reported that living habits including exercise are a critical factor to brain health [28]. There are so many studies showing that living habits or exercises greatly influence the brain. Although it is difficult to specify directly Brain Plasticity on the basis of them, it is considered that they can definitely influence the hypotheses of this study. There are still not many scientific theories and studies. Nevertheless, this study lays the foundation for basic research on the effects of exercises for HRA. In the future, it will be necessary to take into account long-term intervention, specified dependent variables, and the equipment measuring brain changes.

Secondly, HRA being influenced by exercise can be analyzed not only from the physiological aspect (its influence 
on the brain) but from the psychosocial aspect. A previous study showed that doing exercises increased health recognition. Given that, it is possible to look into the relations of exercises with people's health conditions, self-satisfaction, social participation, quality of life, and psychological satisfaction [29].

Thirdly, in regard to the effects of the correction of limited patterns on health, it is possible to increase a degree of freedom in daily living and change behavior more freely from various diseases and action by improving basic functional movements.

In the corrective exercise group, there were significant changes in SFMA scores from baseline to 4 weeks and baseline to 8 weeks. However, this result is the major difference in the statistic between the corrective exercise and the control group. In the corrective exercise group, positive effects of exercise after 4 weeks were maintained after 8 week. However, in the control group, the scores from baseline to 8 weeks were not statistically significant and there is no evidence to show the SFMA score was maintained.

This study tried to find how a corrective exercise program for improving SFMA score and HRA score. This study suggests that the corrective exercise program for improving functional movement pattern and lifestyle behavior was as much effective as general control groups. Following conclusions summarize our findings: 1 . The corrective exercise in the experimental group has positive effect on the SFMA score as well as general squat exercise in the control group. 2. The corrective exercise and general control group has same positive effects on the HRA score. 3. In the corrective exercise group, there was significant difference in SFMA scores from baseline to 4 weeks. 4 . In the corrective exercise group, positive effects of exercise after 4 weeks were maintained after 8 week. This study has a limitation in generalizing the study results because of the small number of study subjects. Corrective exercise intervention with SFMA naturally increased SFMA score. In addition, the intervention was not been carried out completely due to a short experimental period. Further research is needed to increase the generalizability of these findings.

\section{Acknowledgements}

This paper was written by the Academic Publishing Support Project (Character education) for the 110th anniversary of the Sahmyook University.

\section{Conflict of Interest}

The authors declared no potential conflicts of interest with respect to the authorship and/or publication of this article.

\section{References}

1. Im EO, Meleis AI. Meanings of menopause to Korean immigrant women. West J Nurs Res 2000;22:84-102.

2. Bosworth HB, Bastian LA, Rimer BK, Siegler IC. Coping styles and personality domains related to menopausal stress. Womens Health Issues 2003;13:32-8.

3. Kim JH, Noh JC, Kang SJ. Effects of aerobic and resistance exercise on insulin resistance, C-reactive protein, and physical fitness in middle-aged women with metabolic syndrome. Kinemat J 2013;15:81-91.

4. Seo JH, Kim JH, Kim SC. Psychosocial development exercise function: effects of combined exercise on the body composition, blood pressure and atherosclerosis indices in the middle age women pre-hypertension. Korean J Growth Dev 2014;22:37-42.

5. Kim MS, Kim SH, Bang HS. The effect of different set composition according to repetition number during resistance training for 12 weeks on appetite relating hormone, HMW adiponectin, and body composition in the middle-aged obese women. J Korea Entertain Ind Assoc 2014;8:149-57.

6. Yun KY, Kim YJ. The effect of circuit weight training on body composition and physical fitness of middle-aged women for 12 week. J Digit Converg 2016;14:363-70.

7. Park JY. The effects of participation in health exercise program on fitness, health promoting behavior and happiness for adolescents. Korea J Sport 2011;9:245-54.

8. Ko YC, Kim YP. Effects of combined resistance exercise and aerobic exercise course on health-related and energy substrates. J Sport Leis Stud 2012;48:925-36.

9. Kim SY. Effect of health exercise program on insulin resistant and blood lipid of elderly women through convergence. J Digit Converg 2016;14:487-94.

10. Bouchard C, Blair SN, Haskell WL. Physical activity and health. Champaign: Human Kinetics; 2012.

11. Heyward VH, Gibson AL. Advanced fitness assessment and exercise prescription. 7th ed. Champaign: Human Kinetics; 2014.

12. Pollock ML, Wilmore JH, Fox SM. Exercise in health and disease: evaluation and prescription for prevention and rehabilitation. Philadelphia: Saunders; 1984.

13. Yang YJ. Exercise testing and prescription. Hanyang Med Rev 2009;29:20-7.

14. Cook G. Movement: functional movement systems: screening, assessment, corrective strategies. Santa Cruz County: On Target Publications; 2010.

15. Kim YS. Developing a manual for national screening and counseling of people in a transitional period. Health Welf Policy Forum 2008:42-57.

16. Flood A, Chung A, Parker H, Kearns V, O'Sullivan TA. The use of hand grip strength as a predictor of nutrition status in hospital patients. Clin Nutr 2014;33:106-14. 
17. White DK, Neogi T, Nevitt MC, Peloquin CE, Zhu Y, Boudreau $\mathrm{RM}$, et al. Trajectories of gait speed predict mortality in wellfunctioning older adults: the Health, Aging and Body Composition Study. J Gerontol A Biol Sci Med Sci 2013;68:456-64.

18. Grøntved A, Ried-Larsen M, Møller NC, Kristensen PL, Froberg $\mathrm{K}$, Brage S, et al. Muscle strength in youth and cardiovascular risk in young adulthood (the European Youth Heart Study). Br J Sports Med 2015;49:90-4.

19. Lindsay K, Smith $H$. The effect of an exercise therapy program on neuromuscular balance and perceived wellness in sedentary. Paper presented at: Northland Chapter of the American College of Sports Medicine 2015 Annual Meeting; 2015 Nov. Vol. 12: Iss. 1 , Article 10

20. Ahn HK, Lee HJ, Jung DS, Lee SY, Kim SW, Kang JH. The reliability and validity of Korean version of questionnaire for nicotine dependence. J Korean Acad Fam Med 2002;23:999-1008.

21. Joe K, Chai S, Park A, Lee H, Shin I, Min S. Optimum cut-off score for screening of hazardous drinking using the Korean version of alcohol use disorder identification test (AUDIT-K). J Korean Acad Addict Psychiatry 2009;13:34-40.

22. Kinect Australia for the Lifescripts Consortium. Lifescripts practice manual: supporting lifestyle risk factors management in general practice. Canberra: Commonwealth of Australia; 2005.

23. Thomas S, Reading J, Shephard RJ. Revision of the physical activity readiness questionnaire (PAR-Q). Can J Sport Sci 1992;17: 338-45.

24. Kim WY, Cho MS, Lee HS. Development and validation of mini dietary assessment index for Koreans. Korean J Nutr 2003;36: 83-92.

25. Izraelski J. Movement: functional movement systems: screening, assessment, and corrective strategies. J Can Chiropr Assoc 2012;56:158.

26. Glaws KR, Juneau CM, Becker LC, Di Stasi SL, Hewett TE. Intra- and inter-rater reliability of the selective functional movement assessment (sfma). Int J Sports Phys Ther 2014;9:195-207.

27. Small GW, Silverman DH, Siddarth P, Ercoli LM, Miller KJ, Lavretsky H, et al. Effects of a 14-day healthy longevity lifestyle program on cognition and brain function. Am J Geriatr Psychiatry 2006;14:538-45.

28. Walsh R. Lifestyle and mental health. Am Psychol 2011;66:57992.

29. Kang JY, Jee YS, Kim MH, Kim YP. Effects of exercise-participated rate on self-perceived health statusand depression degrees in silver town elderly. Korean J Health Promot 2009;9:114-21. 